\title{
Image reconstruction with noise and error modelling in quantitative photoacoustic tomography
}

\author{
Tanja Tarvainen ${ }^{\mathrm{a}, \mathrm{b}}$, Aki Pulkkinen ${ }^{\mathrm{a}}$, Ben T. Cox ${ }^{\mathrm{c}}$, Jari P. Kaipio ${ }^{\mathrm{a}, \mathrm{d}}$, and Simon R. Arridge ${ }^{\mathrm{b}}$ \\ ${ }^{a}$ Department of Applied Physics, University of Eastern Finland, P.O. Box 1627, FI-70211 \\ Kuopio, Finland \\ ${ }^{\mathrm{b}}$ Department of Computer Science, University College London, Gower Street, London WC1E \\ 6BT, UK \\ 'Department of Medical Physics and Biomedical Engineering, University College London, \\ Gower Street, London WC1E 6BT, UK \\ ${ }^{d}$ Department of Mathematics, University of Auckland, Private Bag 92019, Auckland Mail \\ Centre, Auckland 1142, New Zealand
}

\begin{abstract}
Quantitative photoacoustic tomography is an emerging imaging technique aimed at estimating the optical parameters inside tissue from photoacoustic images. The method proceeds from photoacoustic tomography by taking the estimated initial pressure distributions as data and estimating the absolute values of the optical parameters. Therefore, both the data and the noise of the second (optical) inverse problem are affected by the method applied to solve the first (acoustic) inverse problem. In this work, the Bayesian approach for quantitative photoacoustic tomography is taken. Modelling of noise and errors and incorporating their statistics into the solution of the inverse problem are investigated.
\end{abstract}

Keywords: Photoacoustic tomography, quantitative imaging, inverse problems, Bayesian methods

\section{INTRODUCTION}

Quantitative photoacoustic tomography (QPAT) is an emerging imaging technique aimed at estimating the optical parameters inside tissues from photoacoustic images which are formed by combining optical information and ultrasonic propagation. ${ }^{1}$ Thus, the method proceeds from photoacoustic tomography by taking the estimated initial pressure distributions as data and estimating the absolute values of the optical parameters.

QPAT is a hybrid imaging problem in which the solution of one inverse problem acts as a data for another ill-posed inverse problem. The two inverse problems of QPAT are: 1) reconstruct the initial acoustic pressure distribution from measured acoustic waves and 2) reconstruct the distributions of the optical parameters from the absorbed optical energy density. The first inverse problem 1) is an inverse initial value problem of acoustics. There are a large number of reconstruction techniques available, see e.g. ${ }^{2-4}$ and the references therein. However, in cases in which the speed of sound, acoustic absorption and scattering within the tissue are varying, the inverse problem becomes significantly more challenging. The second inverse problem in QPAT 2) is the optical image reconstruction. In the optical inverse problem the goal is to estimate the concentrations of chromophores. These can be obtained either by directly estimating the chromophore concentrations from photoacoustic images obtained at various wavelengths ${ }^{5-8}$ or by first recovering the absorption coefficients at different wavelengths and then calculating the concentrations from the absorption spectra. ${ }^{5,7,9}$ In order to obtain accurate estimates, scattering effects need to be taken into account. ${ }^{1,10-12}$ As an alternative to the two-step approach to the solution of the QPAT inverse problem, estimation of the optical parameters directly from photoacoustic time-series has also been considered recently. ${ }^{13-18}$

Further author information: (Send correspondence to T.T.)

T.T.: E-mail: tanja.tarvainen@uef.fi, Telephone: +358 403552310

Photons Plus Ultrasound: Imaging and Sensing 2016, edited by Alexander A. Oraevsky, Lihong V. Wang Proc. of SPIE Vol. 9708, 97083Q - (C) 2016 SPIE · CCC code: 1605-7422/16/\$18 · doi: 10.1117/12.2209477 
In this work, the optical inverse problem of QPAT is investigated. Estimation of absorption and scattering at one wavelength is considered. Since in the optical inverse problem of QPAT the data is obtained as a solution of the acoustic inverse problem, both the data and noise are affected by the method applied to solve the acoustic inverse problem. To overcome the problems this causes, modelling of noise and approximation errors in QPAT using a Bayesian framework ${ }^{19}$ was introduced in. ${ }^{20}$ The approach was studied with simulations in diffuse regime. In this work, the approach is tested using data simulated with the radiative transfer equation (RTE) which is a more accurate model for light transport and takes the approach closer to a more realistic scenario.

\section{FORWARD MODEL}

In QPAT, a short (ns) pulse of near-infrared light is used to illuminate the region of tissue of interest. In this work, we use two models for light propagation: the radiative transfer equation (RTE) and the diffusion approximation (DA) of the RTE. ${ }^{21}$ The RTE is a widely accepted model for light transport in tissue. ${ }^{22}$ In QPAT, the time-independent RTE together with a zero-boundary condition is used

$$
\begin{gathered}
\hat{s} \cdot \nabla \phi(r, \hat{s})+\left(\mu_{s}+\mu_{a}\right) \phi(r, \hat{s})=\mu_{s} \int_{S^{n-1}} \Theta\left(\hat{s} \cdot \hat{s}^{\prime}\right) \phi\left(r, \hat{s}^{\prime}\right) \mathrm{d} \hat{s}^{\prime}, \quad r \in \Omega \\
\phi(r, \hat{s})=\left\{\begin{array}{cl}
\phi_{0}(r, \hat{s}), & r \in \epsilon_{j}, \quad \hat{s} \cdot \hat{n}<0 \\
0, & r \in \partial \Omega \backslash \epsilon_{j}, \quad \hat{s} \cdot \hat{n}<0
\end{array}\right.
\end{gathered}
$$

where $\Omega \subset \mathbb{R}^{d}$ is the domain with dimension $d=2$ or 3 and boundary $\partial \Omega, \hat{s} \in S^{d-1}$ is a unit vector in the direction of interest, $\mu_{s}=\mu_{s}(r)$ and $\mu_{a}=\mu_{a}(r)$ are the scattering and absorption coefficients of the medium, respectively, $\phi(r, \hat{s})$ is the radiance, $\hat{n}$ is an outward unit normal, $\phi_{0}(r, \hat{s})$ is a boundary source at a position $\epsilon_{j} \subset \partial \Omega$ and $\Theta\left(\hat{s} \cdot \hat{s}^{\prime}\right)$ is the scattering phase function

$$
\Theta\left(\hat{s} \cdot \hat{s}^{\prime}\right)= \begin{cases}\frac{1}{2 \pi} \frac{1-g^{2}}{\left(1+g^{2}-2 g \hat{s} \cdot \hat{s}^{\prime}\right)}, & d=2 \\ \frac{1}{4 \pi} \frac{1-g g^{2}}{\left(1+g^{2}-2 g \hat{s} \cdot \hat{s}^{\prime}\right)^{3 / 2}}, & d=3\end{cases}
$$

where $g$ is the anisotropy parameter. The RTE has been utilised as light transport model in QPAT e.g. in. ${ }^{11,23,24}$ The DA together with a Robin-type boundary condition is of the form

$$
\begin{aligned}
& -\nabla \cdot \kappa(r) \nabla \Phi(r)+\mu_{a}(r) \Phi(r)=0 \quad r \in \Omega \\
& \Phi(r)+\frac{1}{2 \gamma_{d}} \kappa(r) \frac{\partial \Phi(r)}{\partial \hat{n}}= \begin{cases}\frac{I_{s}}{\gamma_{d}}, & r \in \epsilon_{j} \\
0, & r \in \partial \Omega \backslash \epsilon_{j}\end{cases}
\end{aligned}
$$

where

$$
\Phi(r)=\int_{S^{n-1}} \phi(r, \hat{s}) \mathrm{d} \hat{s}
$$

is the photon fluence, $\kappa(r)=\left(d\left(\mu_{a}(r)+\mu_{s}^{\prime}(r)\right)\right)^{-1}$ is the diffusion coefficient and $\mu_{s}^{\prime}(r)=(1-g) \mu_{s}(r)$ is the reduced scattering coefficient, $I_{s}$ is a diffuse boundary current at the source position $\epsilon_{j} \subset \partial \Omega$, and $\gamma_{d}$ is a dimension-dependent constant which takes values $\gamma_{2}=1 / \pi$ and $\gamma_{3}=1 / 4$. In this work, finite element method (FEM) is used for the numerical solutions of the RTE and DA. ${ }^{11}$

As light propagates within the tissue, it is absorbed by chromophores. The absorbed optical energy density $H(r)$ can be solved from fluence as

$$
H(r)=\mu_{a}(r) \Phi(r)
$$

The light absorption generates localised increases in pressure. The absorbed energy density is connected to the initial acoustic pressure distribution $p_{0}(r)$ through the photoacoustic efficiency which can be identified with the Grüneisen parameter for an absorbing fluid. ${ }^{1}$ In this work, the Grüneisen coefficient is assumed to be a known constant $G$ and the initial acoustic pressure distribution is

$$
p_{0}(r)=p(r, t=0)=G H(r) .
$$


The pressure increase propagates through the tissue as an acoustic wave and is detected by ultrasound sensors on the surface of the object. The propagation of pressure $p(r, t)$ through an acoustically non-absorbing medium is described by wave equation ${ }^{25}$

$$
\nabla^{2} p(r, t)-\frac{1}{c^{2}} \frac{\partial^{2} p(r, t)}{\partial t^{2}}=0
$$

where $c$ is the speed of sound which in this work is assumed to be a known constant. The wave equation (9) is solved together with initial conditions (8) and

$$
\frac{\partial p(r, t=0)}{\partial t}=0 .
$$

A perfectly matched layer (PML) is applied at the boundaries to simulate free space propagation. In this work, the numerical solution of the wave equation is obtained using the $k$-space time-domain method implemented with the k-Wave MATLAB toolbox. ${ }^{26}$

\section{INVERSE PROBLEM}

The inverse problem in QPAT is to solve the distribution of the optical parameters in the medium when the measured pressure on the detectors and the amount of input light are given.

\subsection{Acoustic inverse problem}

The first inverse problem in QPAT is the acoustic inverse initial value problem in which the initial acoustic pressure distribution $p_{0}(r)$ is estimated when the measured acoustic waves $p_{S}$ on the detectors are given. In this work, we use a time reversal method from the k-Wave MATLAB toolbox for the solution of the acoustic inverse problem. ${ }^{26}$ In this approach, the recorded measurements $p_{S}(t)$ are used in time-reversed order as a time-varying Dirichlet boundary condition at the detector positions. The time evolution of the propagating acoustic wave field imposed by the Dirichlet boundary condition is calculated using the wave equation (9) with zero initial conditions. The reconstructed initial pressure $p_{0}$ is then obtained as an acoustic pressure within the domain after time $T$. In this work, the medium is assumed to be non-absorbing and the speed of sound is assumed to be known.

\subsection{Bayesian approach to the optical inverse problem}

The second inverse problem in QPAT is to estimate the optical parameters when the absorbed optical energy density and the input illumination are given. In this work we use the Bayesian approach for the solution of the optical inverse problem and estimate the absorption and scattering distributions within the domain.

A discretised observation model with an additive noise model is of the form

$$
y=A_{h}(x)+e
$$

where $y=\left(H_{1}, \ldots, H_{m}\right)^{\mathrm{T}} \in \mathbb{R}^{m}$ is the measurement vector where $m$ is the number of measurements, $x$ is a discretised parameter distribution and $A_{h}$ is a discretised forward model.

Let us assume that $x$ and $y$ are random variables in finite-dimensional spaces $\mathbb{R}^{N}$ and $\mathbb{R}^{m}$ called parameter and data space respectively. The joint probability density of $x$ and $y$ can be written in terms of conditional probability densities as

$$
\pi(x, y)=\pi(x) \pi(y \mid x)=\pi(y) \pi(x \mid y) .
$$

The solution of the inverse problem is the posterior probability density (Bayes' formula)

$$
\pi(x \mid y) \propto \pi(y \mid x) \pi(x)
$$

where $\pi(x)$ is the prior probability density and $\pi(y \mid x)$ is the likelihood density. ${ }^{19}$ If we assume that the noise $e$ and the unknown $x$ are mutually independent, formula (11) leads to likelihood density

$$
\pi(y \mid x)=\pi_{e}\left(y-A_{h}(x)\right)
$$


where $\pi_{e}$ is the probability distribution of the noise $e$.

If the unknown $x$ and the measurement errors can be modelled as Gaussian random variables, we have

$$
x \sim \mathcal{N}\left(x_{*}, \Gamma_{x}\right), \quad e \sim \mathcal{N}\left(e_{*}, \Gamma_{e}\right)
$$

where $x_{*} \in \mathbb{R}^{N}$ and $e_{*} \in \mathbb{R}^{m}$ are the means and $\Gamma_{x} \in \mathbb{R}^{N \times N}$ and $\Gamma_{e} \in \mathbb{R}^{m \times m}$ are the covariance matrices. In this case, the posterior density (13) becomes

$$
\pi(x \mid y) \propto \exp \left\{-\frac{1}{2}\left(y-A_{h}(x)-e_{*}\right)^{\mathrm{T}} \Gamma_{e}^{-1}\left(y-A_{h}(x)-e_{*}\right)-\frac{1}{2}\left(x-x_{*}\right)^{\mathrm{T}} \Gamma_{x}^{-1}\left(x-x_{*}\right)\right\} .
$$

The practical solution for the inverse problem is obtained by calculating point estimates from the posterior density. Since we are interested in computationally efficient inverse problem solvers, we consider here only the maximum a posteriori (MAP) estimate. It is obtained as

$$
\begin{aligned}
x_{\mathrm{MAP}} & =\underset{x}{\arg \max }\{\pi(x \mid y)\} \\
& =\underset{x}{\arg \min }\left\{\left\|L_{e}\left(y-A_{h}(x)-e_{*}\right)\right\|^{2}+\left\|L_{x}\left(x-x_{*}\right)\right\|^{2}\right\}
\end{aligned}
$$

where Cholesky factorisations of the noise and the prior are $\Gamma_{e}^{-1}=L_{e}^{\mathrm{T}} L_{e}$ and $\Gamma_{x}^{-1}=L_{x}^{\mathrm{T}} L_{x}$, respectively.

Typically in tomographic inverse problems, the mean of the noise is assumed to be zero, $e_{*}=0 \in \mathbb{R}^{m}$, and the covariance is assumed to be a diagonal matrix with known (constant) variance $\sigma$, that is $\Gamma_{e}=\Gamma_{\sigma}=\operatorname{diag}\left(\sigma^{2}\right) \in$ $\mathbb{R}^{\mathrm{m} \times \mathrm{m}}$. In this case, the MAP estimate is obtained as

$$
x_{\mathrm{MAP}}=\underset{x}{\arg \min }\left\{\left\|1 / \sigma\left(y-A_{h}(x)\right)\right\|^{2}+\left\|L_{x}\left(x-x_{*}\right)\right\|^{2}\right\} .
$$

which we refer as the MAP estimate with the conventional noise model (MAP-CNM).

In QPAT, the solution method of the acoustic inverse problem affects how the noise of the time-domain acoustic data is transferred to the optical problem. Therefore, the noise of the optical inverse problem is necessarily not uncorrelated and it may have a non-zero mean. A more accurate noise model can be, for example, approximated as follows. ${ }^{20}$ First a set of noise samples of pressure measurements are simulated. Then, the inverse initial value problem is solved using these noise samples as data. As a result, noise samples of the optical inverse problem $e^{(l)}$ are obtained. The mean and the covariance of the noise model can then be approximated using these noise samples as

$$
\begin{aligned}
e_{*} & =\frac{1}{L} \sum_{l=1}^{L} e^{(l)} \\
\Gamma_{e} & =\frac{1}{L-1} \sum_{l=1}^{L} e^{(l)} e^{(l) \mathrm{T}}-e_{*} e_{*}^{\mathrm{T}}
\end{aligned}
$$

where $L$ is the number of samples. These are then applied in the solution of the minimisation problem (16). In the following sections, we refer to the solution of (16) together with (18)-(19) as the MAP estimate with an approximate noise model (MAP-ANM).

In practise, the numerical implementation of the acoustic inverse method also affects the measured data. Thus, the optical energy density distribution obtained as the solution of the acoustic inverse problem contains modelling error $\varepsilon$ which can be due to e.g. discretisation of the geometry and time, implementation of the boundary conditions and smoothing of the data by the acoustic solver. Therefore, let us write the observation model (11) in the form

$$
\begin{aligned}
y & =A_{h}(x)+e \\
y_{h}+\left(y-y_{h}\right) & =A_{h}(x)+e \\
y_{h} & =A_{h}(x)+\varepsilon+e
\end{aligned}
$$


where $\varepsilon=y_{h}-y$ is the modelling error of the acoustic solver which describes the discrepancy between the "ideal" data $y$ and data containing errors due to the acoustic solver $y_{h}$. Then, similarly as in the framework of Bayesian approximation error modelling, ${ }^{19}$ a Gaussian approximation is constructed for $\varepsilon$, and the total error $n=\varepsilon+e$ is approximated by a Gaussian distribution, thus

$$
\varepsilon \sim \mathcal{N}\left(\varepsilon_{*}, \Gamma_{\varepsilon}\right), \quad n \sim \mathcal{N}\left(n_{*}, \Gamma_{n}\right) .
$$

where $n_{*}=\varepsilon_{*}+e_{*}$ and $\Gamma_{n}=\Gamma_{\varepsilon}+\Gamma_{e}$. Furthermore, the mutual dependence of $x$ and $\varepsilon$ is ignored. The MAP estimate with the noise and error modelling (MAP-AEM) is obtained as

$$
x_{\mathrm{MAP}}=\underset{x}{\arg \min }\left\{\left\|L_{n}\left(y-A_{h}(x)-n_{*}\right)\right\|^{2}+\left\|L_{x}\left(x-x_{*}\right)\right\|^{2}\right\}
$$

where $\Gamma_{n}^{-1}=L_{n}^{\mathrm{T}} L_{n}$.

\section{SIMULATIONS}

The Bayesian approach for the solution of the optical inverse problem of QPAT was tested with simulations. MAP estimates were computed from simulated data utilising different noise models and error modelling.

A rectangular domain $\Omega$ of size $20 \mathrm{~mm} \times 20 \mathrm{~mm}$ was considered. The Grüneisen coefficient was assumed to be a known constant with value $G=1$. Four illuminations, one edge working as a light source in turn, were considered.

Two datasets using either the RTE or the DA as light transport model were simulated. Absorption and scattering distributions shown in the left columns of Figs. 1 and 2 were studied. The anisotropy parameter was $g=0.8$ throughout the domain. The absorption and scattering values were represented in piecewise constant bases using $K=16796$ triangular elements. The optical forward problem was solved using the RTE (1)-(2) and the DA (4)-(5). The FE-discretisation consisted of 16796 triangular elements and 8559 nodes. Then, for both datasets, the absorbed optical energy density distribution and the initial pressure were computed using (7) and (8), respectively, and the $k$-space method was used to simulate propagation of the pressure wave though the domain. The discretisation of the computation domain consisted of $1024 \times 1024$ pixels. The time-varying acoustic pressure was recored at 316 sensors located around the target and noise with a standard deviation of $1 \%$ of the peak amplitude of the simulated pressure signal was added to the datasets. In order to obtain the data for the optical inverse problem, the acoustic inverse problem was solved using the time reversal method. The acoustic inverse problem was solved using both datasets in discretisation of $128 \times 128$ pixels. The absorbed optical energy density in the reconstruction grid, which is the data of the optical inverse problem, was computed from the initial pressure using (8) with Grüneisen coefficient $G=1$.

The MAP estimates using the three approaches were computed by minimising (17) (MAP-CNM), (16) (MAPANM) and (21) (MAP-AEM) using a Gauss-Newton method equipped with a line search algorithm and positivity constraint. The prior distribution utilised was the informative smoothness prior $\pi(x)^{27,28}$ with correlation length $4 \mathrm{~mm}$ for both absorption and scattering, means $\eta_{\mu_{a}}=0.01$ and $\eta_{\mu_{s}}=1$, and standard deviations for the background $\sigma_{\mu_{a}}^{\mathrm{bg}}=0.002, \sigma_{\mu_{s}}^{\mathrm{bg}}=0.2$ and the inclusions $\sigma_{\mu_{a}}^{\text {incl }}=0.01$ and $\sigma_{\mu_{s}}^{\text {incl }}=1$. For the MAP estimate with the conventional noise model (17), the noise was considered to be uncorrelated Gaussian distributed noise with zero mean. The standard deviation $\sigma$ of the noise was set as $1 \%$ of the peak amplitude of the simulated data. For the MAP estimate with the approximate noise model (16), a simulated noise statistics (18)-(19) which was computed by simulating and reconstructing acoustic noise samples was used, and for the modelling error, the approximation errors were simulated as $\mathrm{in}^{20}$ using the DA as a light transport model.

The reconstructed absorption and scattering distributions from the RTE simulated data are shown in Fig. 1 and from the DA simulated data are shown in Fig. 2. The calculated mean relative errors

$$
\bar{E}_{\mu_{a}}=100 \% \cdot \frac{\left\|\mu_{a}-\hat{\mu}_{a}\right\|}{\left\|\mu_{a}\right\|}, \quad \bar{E}_{\mu_{s}}=100 \% \cdot \frac{\left\|\mu_{s}-\hat{\mu}_{s}\right\|}{\left\|\mu_{s}\right\|}
$$

where $\mu_{a}$ and $\mu_{s}$ are the simulated absorption and scattering distributions interpolated to the solution space and $\hat{\mu}_{a}$ and $\hat{\mu}_{s}$ are the estimated values for both datasets are given in Table 1 . 


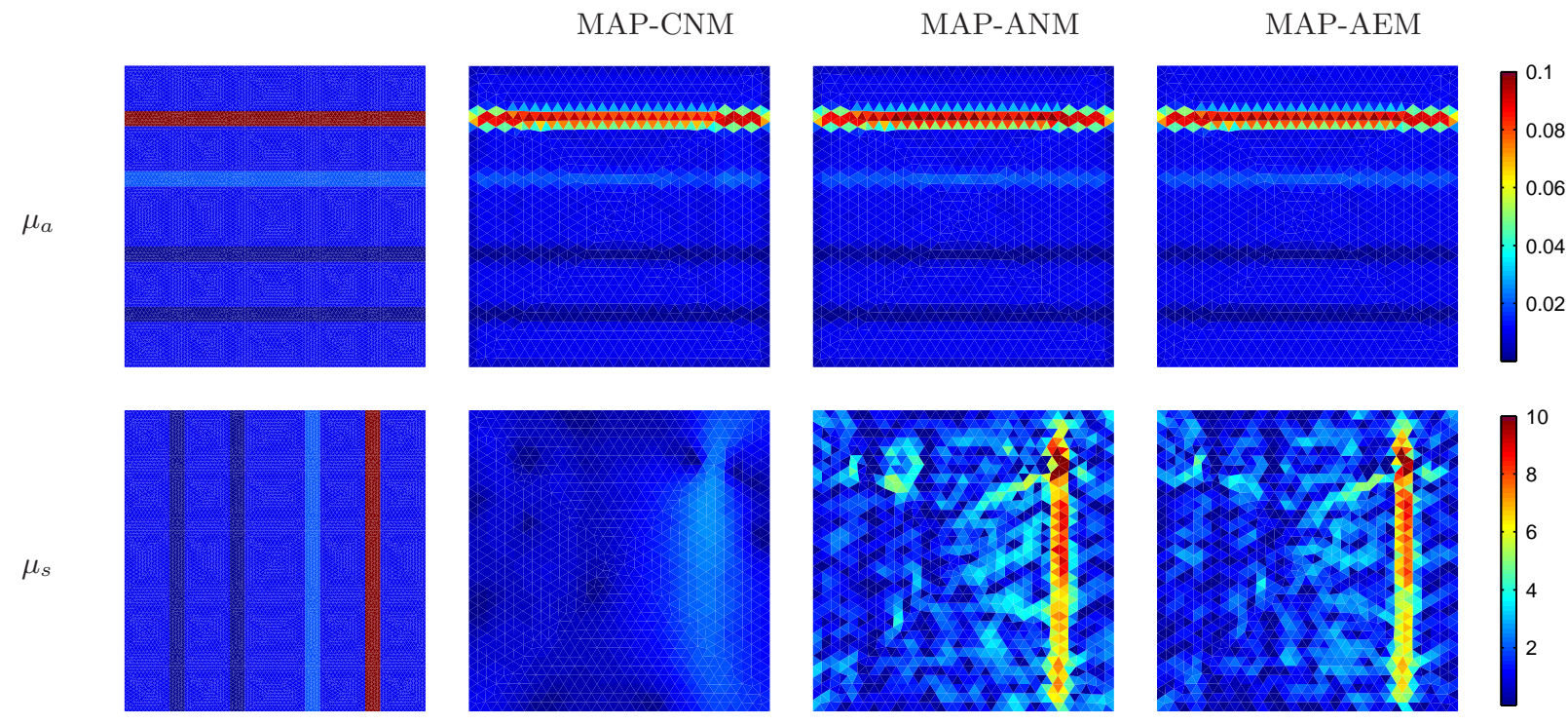

Figure 1. Reconstructed absorption (top row) and scattering (bottom row) distributions from RTE simulated data. Images from left to right: simulated distributions (first column), reconstructions obtained with the conventional noise model MAP-CNM (second column), approximate noise model MAP-ANM (third column) and approximate noise model and error modelling MAP-AEM (fourth column). The units of the colorbars are in $\mathrm{mm}^{-1}$.

Table 1. Mean relative errors of absorption and scattering MAP estimates $\bar{E}_{\mu_{a}}$ and $\bar{E}_{\mu_{s}}$, respectively, obtained with noise models MAP-CNM (17), MAP-ANM (16), (18)-(19), and error model MAP-AEM (21) for RTE and DA simulated data.

\begin{tabular}{cccccc}
\hline \hline & \multicolumn{3}{c}{$\mathrm{RTE}$} & & \multicolumn{2}{c}{$\mathrm{DA}$} \\
\cline { 2 - 3 } \cline { 5 - 6 } & $\bar{E}_{\mu_{a}}(\%)$ & $\bar{E}_{\mu_{s}}(\%)$ & & $\bar{E}_{\mu_{a}}(\%)$ & $\bar{E}_{\mu_{s}}(\%)$ \\
\hline MAP-CNM & 17 & 72 & & 16 & 71 \\
MAP-ANM & 15 & 59 & & 16 & 48 \\
MAP-AEM & 14 & 54 & & 14 & 56 \\
\hline \hline
\end{tabular}

The results show that noise and error modelling improve absorption and scattering estimates both qualitatively and quantitatively. The improvement is more clear with scattering since the problem is more ill-posed for estimating scattering. The estimates from the RTE simulated data and the DA simulated data are equally good, although the DA was used for computing the modelling error statistics.

\section{CONCLUSIONS}

In this work, the optical inverse problem of QPAT was considered. Modelling of noise and errors was studied in the framework of Bayesian inversion using data simulated with the RTE and reconstructions computed using the DA. The results show that modelling of the noise and the approximation errors can improve the optical reconstructions of QPAT by improving the accuracy of the estimated parameters and by removing systematic artefacts caused by the acoustic solver also in a more realistic case in which the data is simulated using a more accurate light transport model. To further improve, modelling of the errors caused by using the DA as the light transport model (instead of the more accurate RTE) and model reduction could be investigated by Bayesian approximation error modelling. ${ }^{19}$ 


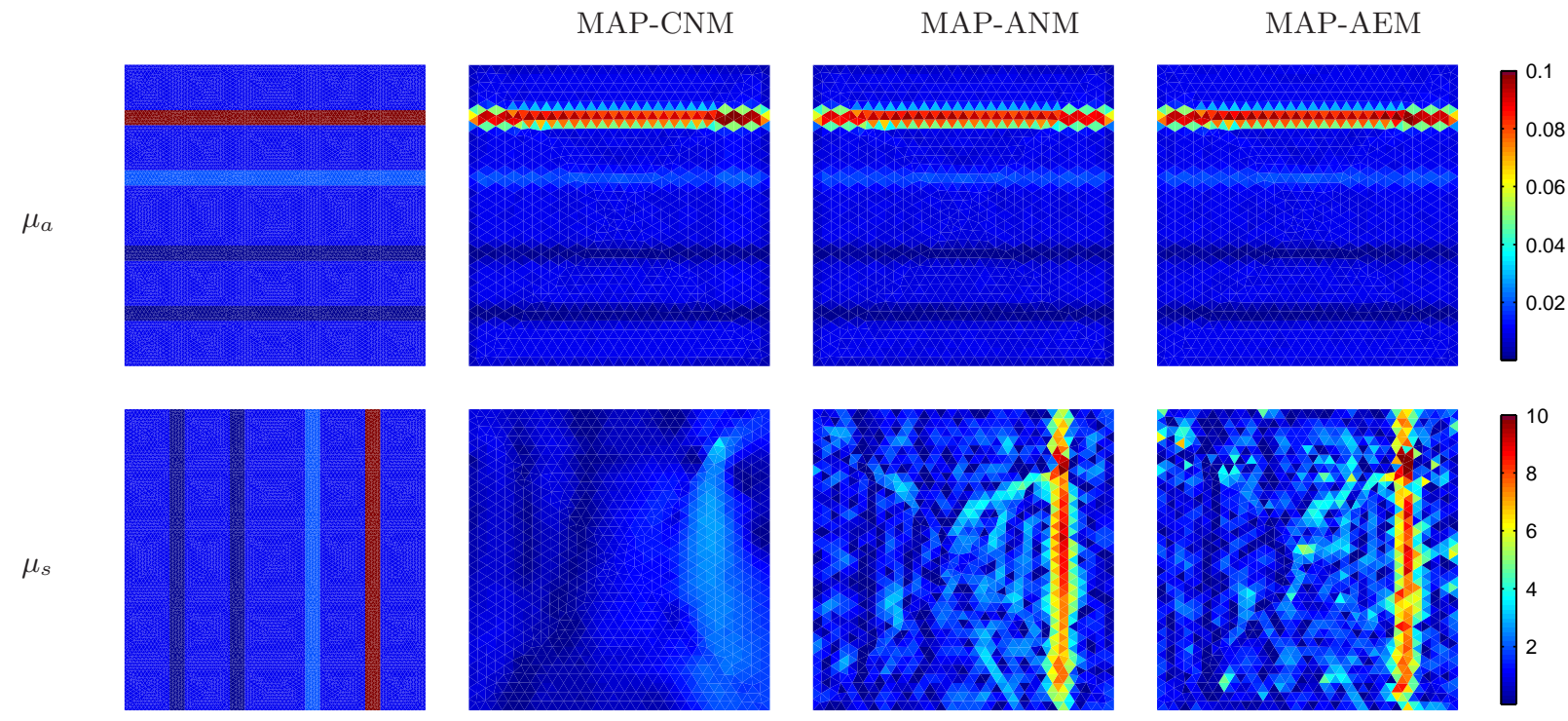

Figure 2. Reconstructed absorption (top row) and scattering (bottom row) distributions from DA simulated data. Images from left to right: simulated distributions (first column), reconstructions obtained with the conventional noise model MAP-CNM (second column), approximate noise model MAP-ANM (third column) and approximate noise model and error modelling MAP-AEM (fourth column). The units of the colorbars are in $\mathrm{mm}^{-1}$.

\section{ACKNOWLEDGMENTS}

This work has been supported by the Academy of Finland (projects 136220, 272803, 286247, and 250215 Finnish Centre of Excellence in Inverse Problems Research).

\section{REFERENCES}

[1] Cox, B., Laufer, J. G., Arridge, S. R., and Beard, P. C., "Quantitative spectroscopic photoacoustic imgaging: a review," J Biomed Opt 17(6), 061202 (2012).

[2] Xu, M. and Wang, L. V., "Photoacoustic imaging in biomedicine," Rev Sci Instrum 77, 041101 (2006).

[3] Kuchment, P. and Kunyansky, L., "Mathematics of thermoacoustic tomography," European Journal of Applied Mathematics 19, 191-224 (2008).

[4] Wang, L. V., ed., [Photoacoustic Imaging and Spectroscopy], CRC Press (2009).

[5] Cox, B. T., R., S., Arridge, and Beard, P. C., "Estimating chromophore distributions from multiwavelength photoacoustic images," J Opt Soc Am A 26(2), 443-455 (2009).

[6] Laufer, J., Cox, B., Zhang, E., and Beard, P., "Quantitative determination of chromophore concentrations form 2D photoacoustic images using a nonlinear model-based inversion scheme," Appl Opt 49(8), 1219-1233 (2010).

[7] Bal, G. and Ren, K., "On multi-spectral quantitative photoacoustic tomography in a diffusive regime," Inv Probl 28, 025010 (2012).

[8] Pulkkinen, A., Cox, B. T., Arridge, S. R., Kaipio, J. P., and Tarvainen, T., "A Bayesian approach to spectral quantitative photoacoustic tomography," Inv Probl 30, 065012 (2014).

[9] Razansky, D., Baeten, J., and Ntziachristos, V., "Sensitivity of molecular target detection by multispectral optoacoustic tomography (MSOT)," Med Phys 36(3), 939-945 (2009).

[10] Bal, G. and Ren, K., "Multi-source quantitative photoacoustic tomography in a diffusive regime," Inv Probl 27, 075003 (2011). 
[11] Tarvainen, T., Cox, B. T., Kaipio, J. P., and Arridge, S. R., "Reconstructing absorption and scattering distributions in quantitative photoacoustic tomography," Inv Probl 28, 084009 (2012).

[12] Pulkkinen, A., Kolehmainen, V., Kaipio, J. P., Cox, B. T., Arridge, S. R., and Tarvainen, T., "Approximate marginalization of unknown scattering in quantitative photoacoustic tomography," Inv Probl Imag 8(3), 811-829 (2014).

[13] Shao, P., Harrison, T., and Zemp, R. J., "Iterative algorithm for multiple illumination photoacoustic tomography (MIPAT) using ultrasound channel data," Biomed Opt Express 3(12), 3240-3248 (2012).

[14] Song, N., Deumié, C., and Silva, A. D., "Considering sources and detectors distributions for quantitative photoacoustic tomography," Biomed Opt Express 5(11), 3960-3974 (2014).

[15] Haltmeier, M., Neumann, L., and Rabanser, S., "Single-stage reconstruction algorithm for quantitative photoacoustic tomography," Inv Probl 31, 065005 (2015).

[16] Gao, H., Feng, J., and Song, L., "Limited-view multi-source quantitative photoacoustic tomography," Inv Probl 31, 065004 (2015).

[17] Ding, T., Ren, K., and Valleélian, S., "A one-step reconstruction algorithm for quantitative photoacoustic imaging," Inv Probl 31, 095005 (2015).

[18] Pulkkinen, A., Cox, B. T., Arridge, S. R., Kaipio, J. P., and Tarvainen, T., "Direct estimation of optical parameters from photoacoustic time series in quantitative photoacoustic tomography," IEEE Trans Med Imag . Submitted.

[19] Kaipio, J. and Somersalo, E., [Statistical and Computational Inverse Problems], Springer, New York (2005).

[20] Tarvainen, T., Pulkkinen, A., Cox, B. T., Kaipio, J. P., and Arridge, S. R., "Bayesian image reconstruction in quantitative photoacoustic tomography," IEEE Trans Med Imag 32(12), 2287-2298 (2013).

[21] Arridge, S. R., "Optical tomography in medical imaging," Inv Probl 15, R41-R93 (1999).

[22] Ishimaru, A., [Wave Propagation and Scattering in Random Media], vol. 1, Academic Press, New York (1978).

[23] Saratoon, T., Tarvainen, T., Cox, B. T., and Arridge, S. R., "A gradient-based method for quantitative photoacoustic tomography using the radiative transfer equation," Inv Probl 29, 075006 (2013).

[24] Mamonov, A. V. and Ren, K., "Quantitative photoacoustic imaging in radiative transport regime," Comm. Math. Sci 12, 201-34 (2014).

[25] Cox, B. T. and Beard, P. C., "Fast calculation of pulsed photoacoustic fields in fluids using $k$-space methods," J Acoust Soc Am 117(6), 3616-3627 (2005).

[26] Treeby, B. E. and Cox, B. T., "k-Wave: MATLAB toolbox for the simulation and reconstruction of photoacoustic wave fields," J Biomed Opt 15(2), 021314 (2010).

[27] Arridge, S. R., Kaipio, J. P., Kolehmainen, V., Schweiger, M., Somersalo, E., Tarvainen, T., and Vauhkonen, M., "Approximation errors and model reduction with an application in optical diffusion tomography," Inv Probl 22, 175-195 (2006).

[28] Kolehmainen, V., Schweiger, M., Nissilä, I., Tarvainen, T., Arridge, S. R., and Kaipio, J. P., "Approximation errors and model reduction in three-dimensional diffuse optical tomography," J Opt Soc Am A 26(10), 22572268 (2009). 